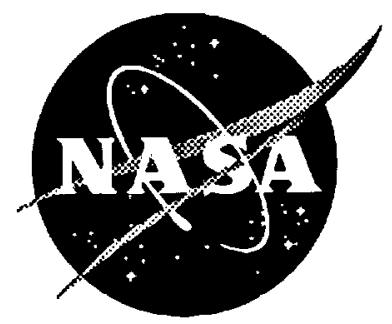

\title{
Design Oriented Structural Analysis
}

Gary L. Giles

Langley Research Center, Hampton, Virginia

(NASA-TM-109124) DESIGN ORIENTED

N94-34722

STRUCTURAL ANALYSIS (NASA. LaNgleY

Research (enter) $12 \mathrm{p}$

Unclas

$63 / 390012106$

June 1994

National Aeronautics and

Space Administration

Langley Research Center

Hampton, Virginia 23681-0001 
$\cdots$

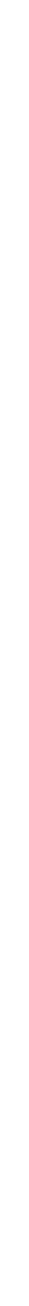

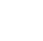




\title{
DESIGN ORIENTED STRUCTURAL ANALYSIS
}

\author{
Gary L. Giles \\ NASA Langley Research Center \\ Hampton, Virginia 23681
}

\begin{abstract}
Desirable characteristics and benefits of design oriented analysis methods are described and illustrated by presenting a synoptic description of the development and uses of the Equivalent Laminated Plate Solution (ELAPS) computer code. ELAPS is a design oriented structural analysis method which is intended for use in the early design of aircraft wing structures. Model preparation is minimized by using a few large plate segments to model the wing box structure. Computational efficiency is achieved by using a limited number of global displacement functions that encompass all segments over the wing planform. Coupling with other codes is facilitated since the output quantities such as deflections and stresses are calculated as continuous functions over the plate segments. Various aspects of the ELAPS development are discussed including the analytical formulation, verification of results by comparison with finite element analysis results, coupling with other codes, and calculation of sensitivity derivatives. The effectiveness of ELAPS for multidisciplinary design application is illustrated by describing its use in design studies of high speed civil transport wing structures.
\end{abstract}

\section{Introduction}

The design process for aerospace vehicles progresses through conceptual, preliminary and detailed design phases in which the sophistication of the analysis methods and the quality of the design data increases. During conceptual design, many alternative configurations are evaluated to determine the general characteristics (e.g., type and number of engines), the size (gross weight) and shape (external geometry parameters) of a candidate configuration which will best meet a specified measure of overall vehicle performance. A representative configuration sizing code for use in conceptual design is the Flight Optimization System (FLOPS) [1] which operates on such system-level design variables. Typically in such codes, use is made of relatively simple, experience-based equations or elementary analytical models to relate these system design variables to the important vehicle characteristics from each engineering discipline such as aerodynamic lift and drag and structural weight. More detailed disciplinary-level data associated with the physics of the vehicle design problem such as pressures in the external flowfield or stresses and strains in the airframe structure are usually not calculated in the conceptual design phase. Rather, it is assumed that all design requirements and interdisciplinary interactions associated with the disciplinary-level data have been satisfied and are contained implicitly in the system-level data which underlies the experience-based equations. However, such equations should not be used beyond their limits of validity

In preliminary design, a baseline design is established and refined using explicit, physics-based analysis methods which explicitly relate vehicle response to the discipline-level design variables. During detailed design, the final configuration is verified using sophisticated analyses and refined analytical models. In general, the process is characterized by an increasing level of refinement and detail in the analysis methods and models and a continual reduction in the number of design alternatives as design variables are frozen until the final design is established

During conceptual and early preliminary design, multidisciplinary design trades are performed to evaluate the system-level variables which have the greatest potential for improving the vehicle performance. However, if implicit, experience-based design data as opposed to explicit, physicsbased data is used, the design may have to be modified during detailed design if deficiencies are uncovered as a result of more in-depth analyses. Such design modifications, can have a major negative impact on the cost of the product. Hence, there is a continuing need to improve the quality of the design data in a timely manner through use of explicit, physics-based analysis methods early in the design process

A class of techniques denoted design oriented analysis methods are intended to generate such data and are recognized as key components for developing multidisciplinary design optimization (MDO) capabilities [2]. Significant improvement in capabilities for generating design data has been achieved by simply implementing existing, sophisticated analysis codes and procedures on new and emerging computer hardware, ranging from engineering workstations to computers with massively parallel processors. However, even with these faster analysis tools, the design cycle is still relatively time-consuming and costly. For the design of structures, improvements are needed to reduce time for preparation of analytical models, application of loads and repetitive analysis during resizing of structural members. An early example of the development of a design oriented method for structures is the TSO (Aeroelastic Tailoring and Structural Optimization) computer program.[3,4] This code has had widespread use for aeroelastic tailoring of composite wings [5]. However, the structural formulation used in TSO is limited to trapezoidal planforms.

In this paper, desirable characteristics and benefits of design oriented analysis methods will be illustrated by presenting a synoptic description of the development and uses of the ELAPS (Equivalent Laminated Plate Solution) computer code. ELAPS was developed to model and analyze aircraft wing structures with general planforms early in the design process 
[6,7]. Various aspects of the ELAPS development will be discussed including the analytical formulation, verification of results by comparison with finite element analysis results, coupling with other codes, and calculation of sensitivity derivatives. The effectiveness of ELAPS for multidisciplinary design application is illustrated by describing its use in design studies of high speed civil transport (HSCT) wing structures.

A comprehensive review of design oriented structural analysis methods is not attempted. Rather, the intent of this paper is to illustrate the advantages of design oriented analysis methods by summarizing the ELAPS-related developments as an overview presentation. Technical details of the methods and procedures that are discussed are provided in referenced publications.

\section{Design Oriented Analysis}

Design oriented analysis methods are intended to provide the capability for rapid and reliable assessment of the effects of proposed design changes. The desirable characteristics of design oriented analysis methods (shown in italics) are summarized in this section. Discussions of each characteristic is given in generic terms that are applicable to such methods from any discipline. However, these characteristics are illustrated for the structures discipline in subsequent sections by presenting a synopsis of the development and use of the ELAPS equivalent plate structural analysis code.

Adequate accuracy for early preliminary design must be provided by the method in order to reliably represent disciplinary behavior in system trade studies. Such a method must be based on the principles of physics which describe the important phenomenon and responses for the discipline being investigated. It is essential that the method provide correct trend data (relative changes in response resulting from changes in design variables) in order to insure that the design progresses in the proper direction. In this process, high levels of accuracy at intermediate points (sufficiently removed from the final design) may not be necessary. If increased accuracy is needed, the opportunity exists to scale the approximate results from a design oriented analysis in order to reflect the increased accuracy of high fidelity analysis methods. Such scaling is discussed in a later section of the paper.

Efficient computation is important because design oriented analysis methods are used repetitively to investigate a large number of design alternatives. Various approximations and simplifications in the underlying theory are used to achieve computational efficiency. Computer code used to implement the method should facilitate taking advantage of the speed provided by advanced computer hardware.

Capability to trade accuracy for speed can be provided with user selected levels of analytical modeling by controlling which terms are included in the underlying theory and/or controlling the level of refinement of a discretized model. Generally, such control is reflected by the total number of unknowns in the resulting set of governing equations

Minimal time for model preparation and modification can be achieved by using a simplified analytical model required by an approximate analysis method or by developing automated procedures to generate high fidelity analytical models. The geometric description (often a large amount of data) associated with such models should be governed by a relatively small number of parameters which can be used as design variables. Such a parameterized geometric description can be used directly for modifying a model to reflect changes in values of design variables

Capability to generate sensitivity derivatives of outputs from a design oriented analysis code with respect to a set of design variables that are adjusted by a designer or a nonlinear programming code to achieve some optimal design objective is a key technology. Alternative approaches for calculating such derivatives include finite differences, analytical differentiation of governing equations and automatic differentiation. Automatic differentiation is based on a pre-compiler which computes derivatives of all elementary operations contained in each line of the original analysis code and applies the chain rule over and over again to compute the desired derivative information. References to these approaches are cited in a later section which provides a brief description of each approach along with a discussion of their benefits and shortcomings.

Ease of coupling with other codes is important since design oriented analysis methods are often used in multidisciplinary analysis and/or multidisciplinary optimization systems. An example is aeroelastic calculations in which methods from the aerodynamics and structures disciplines are coupled. Analysis codes from different disciplines usually have different analysis grids on which information is calculated. It is desirable that a design oriented analysis be formulated in a way that eliminates or simplifies the cumbersome procedures that are often used to transform data between the non-matching grids.

The combination of these features in a design oriented analysis code provides a capability which can be effective for use in multidisciplinary design optimization systems for performing design studies during early phases of design. Such integrated systems offer significant potential to achieve the benefits of reducing design cycle time and/or resulting in an improved product design.

\section{Equivalent Laminated Plate Solution (ELAPS)}

\subsection{Background}

Airframe weight is the key structural parameter used in aircraft system studies. In conceptual and early preliminary design, empirical weight equations are often used to calculate this data. These equations are principally functions of the vehicle external geometry and require very little information about the internal structural details. In later design phases, the locations and sizes of an assemblage of structural members making up the airframe are determined. The airframe should be lightweight but also have sufficient strength and stiffness necessary to satisfy all the requirements throughout its flight envelope. General purpose finite element structural analysis codes are available to model and analyze the static and dynamic response of airframes in great detail. However, such analyses usually require considerable calendar time to generate the finite element model and repetitive analyses can be computationally expensive

Design oriented structural analysis methods have been developed to bridge the gap between weight equations and detailed finite element analyses. An early example of such a capability is the equivalent plate model of the wing structure which is used in the aeroelastic tailoring and structural optimization (TSO) computer code. This code has had widespread use for aeroelastic tailoring of composite wings. However, the structural formulation used in TSO is limited to trapezoidal planforms.

The ELAPS code was developed to provide a more general equivalent plate representation. Wing structures with general 
planform geometry such as cranked wing boxes can be modeled. Also, the model can contain unsymmetric wing cross sections which can arise from airfoil camber or from having different thicknesses in the upper and lower cover skins. This structural analysis method can be used to calculate static deflections and stresses and vibration frequencies and modes. ELAPS contains many of the desirable characteristics for a design oriented analysis code and these features will be highlighted in the following description of its development and use.

\subsection{Analytical Formulation}

A wing box structure is represented as an equivalent plate in this formulation. Planform geometry of this equivalent plate is defined by multiple trapezoidal segments as illustrated by the two-segment box in Figure 1. Each plate segment has upper and lower cover skins which may contain multiple layers of composite material. The cross-sectional view of a typical segment illustrates the generality to define out-of-plane shapes such as the twist and camber characteristics of an aircraft wing. The cross-sectional dimensions of wing depth, camber definition and cover skin thicknesses are defined by polynomials which vary over the planform of each segment. For static analysis, loading is applied to the wing box as concentrated forces or distributed loads. Mass properties for dynamic analysis are defined by concentrated or distributed quantities.

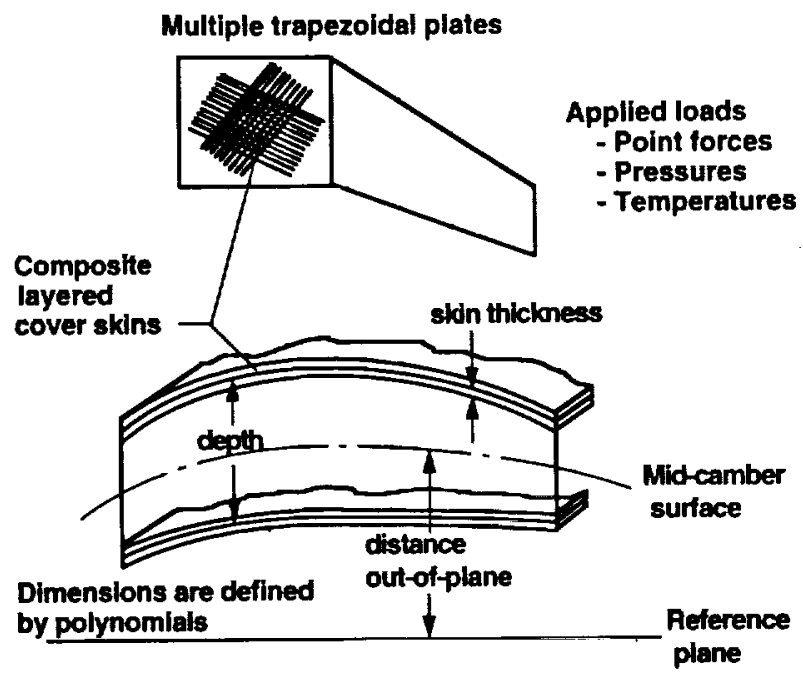

Figure 1. Analytical model for equivalent plate analysis

The specification of model characteristics as continuous distributions in polynomial form on only a few members requires only a small fraction of the volume of input data for a corresponding finite element structural model where geometry and stiffness properties are specified at discrete locations. The resulting reduction in model preparation time is important during early design phases when many candidate configurations are being assessed. Also, the geometric locations of the mass quantities and the applied loadings can be independently defined, i.e., they are not referenced to a set of joint locations as in a finite element model. The ease of relocating these quantities without disupting other aspects of the model is important during early design when such changes often occur. Finally, the polynomial description of model characteristics lends itself to use with optimization algorithms since the polynomial coefficients can be used directly as design variables. Therefore, the analytical formulation in ELAPS requires minimal time for model preparation and modification.

The analysis procedure, outlined in Figure 2, is based on the Ritz method in which the deflection of the structure is described by assumed polynomial displacement functions. In order to achieve a high level of computational efficiency, the displacement functions $U, V$ and $W$ are defined by combinations of terms from a power series in the chordwise coordinate $x$ and a power series in the spanwise coordinate $y$. as indicated in the figure. These $x$ and $y$ coordinates are nondimensionalized by dividing by a reference length (usually the wing semispan) so that the coefficients all have the same units of length. Substituting these functions into the expression for total energy and differentiating to minimize the energy produces a set of linear, simultaneous equations which can solved for the desired set of unknown polynomial coefficients.

\section{Filt solution technique used}

Polynomial displacement functions

$$
\begin{aligned}
& U=\sum_{j} \sum A_{i j} x^{I} y^{j} \\
& v=\sum_{k} \sum_{l} \mathbf{q}_{\mathbf{k} !} x^{k} y^{1}
\end{aligned}
$$

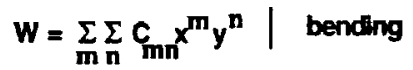

Minimization of energy

$$
\Omega=1 / 2 \int_{\mathrm{vol}}\left(\varepsilon^{T_{Q}} \varepsilon-2 \Delta T \alpha^{T} Q \varepsilon\right) d V
$$

Solve equations for polynomial coefficlents; A, B and C

$$
\left[\begin{array}{lll}
K_{u u} & K_{u v} & K_{u w} \\
K_{u v} & K_{v v} & K_{v w} \\
K_{u w} & K_{v w} & K_{w w}
\end{array}\right]\left\{\begin{array}{l}
A_{i j} \\
B_{k l} \\
C_{m n}
\end{array}\right\}=\left\{\begin{array}{l}
P_{u} \\
P_{v} \\
P_{w}
\end{array}\right\}
$$

Figure 2. Outline of equivalent plate analysis procedure.

These coefficients are used to calculate deflections, strains and stresses over the planform of the plate segments. Typically, the number of terms in the polynomial displacement functions is relatively small (around a hundred) and results in efficient computation. The number of terms is selected by the user. In this manner, the user is given the capability to trade accuracy for speed. However, there are upper limits on the degree of the polynomials that can be specified for the displacement functions. This limit results from using power series terms which are non-orthogonal. High-degree terms produce nearly linearly dependent equations and cause the set of governing equations to become ill-conditioned. Typical, practical upper limits on the power series terms are fourth degree in $x$ and seventh degree in $y$.

For static analysis, rigid-body motion of the equivalent plate must be constrained. These constraints are often referred to as boundary conditions. Two methods are used to apply the constraints for static analysis. In the first method, selected terms are excluded from the displacement functions in order to specify that displacements or slopes are zero along the $x$-axis, which is usually located at the aircraft centerline. For example, a clamped boundary condition along the $x$-axis could be 
specified omitting all terms containing $y$ to the zero power $y^{O}$ and all terms containing $y$ to the first power $y^{l}$ from the expression for transverse bending, $W$. In the second method, stiff springs are used to constrain the translations and/or rotations at selected locations. Although displacements cannot be specified to be exactly zero at a selected location, use of sufficiently stiff springs will provide a good approximation to the desired condition. In addition, an eigenvalue shift parameter has been included for use in vibration analysis. This shift parameter allows a vibration analysis to be performed on a model with unconstrained (rigid-body) motions

\subsection{Verification}

Equivalent plate results have been compared to conventional finite element results to verify the accuracy and to determine the relative computational times. Initial applications were representative of fighter aircraft wings which were relatively thin and stiff because of the high speeds and high maneuver load factors at which they were intended to operate. In such wings, bending is the primary contributor to wing deflection and the secondary effects of transverse shear deflection can be ignored. ELAPS is well-suited to analyze such wings since its formulation assumed that the wing has infinite shear stiffness. A representative example of a fighter wing structure was studied in Reference [7] to assess the accuracy and computational efficiency of ELAPS. Selected results from that study are presented in this section to illustrate typical levels of accuracy which can be expected. The planforms of the finite element model and equivalent plate model used in the study are shown in Figure 3. The planforms are composed of a clippeddel ta outer segment with a $\mathbf{4 5}$ degree leading edge sweep and an inner segment to represent a carry through structure.

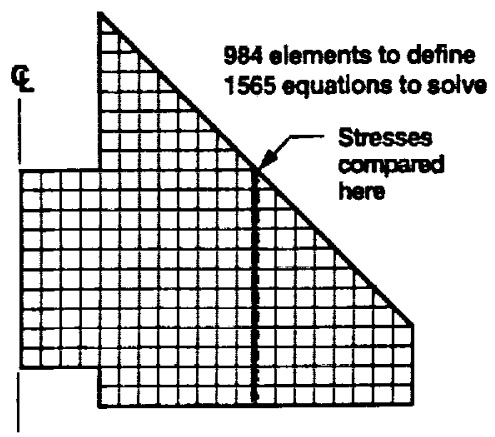

(a) Finite element model

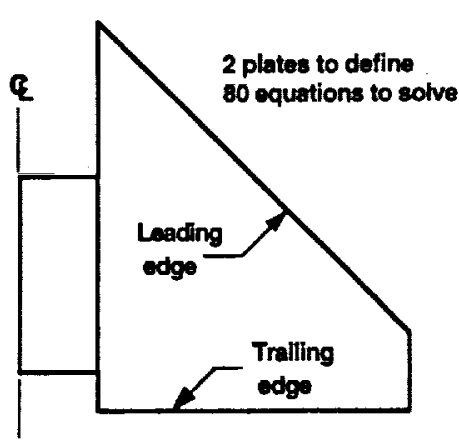

(b) Equivalent plate model

Figure 3. Example structural models used for verification study.
The deflections along the leading and trailing edges of the outer segment resulting from a thermal loading differed by approximately $5 \%$ between the two models as shown in Figure 4. Comparison of the lowest 10 natural vibration frequencies were also shown in the study to differ in the $5 \%$ range. This level of accuracy has been found to be typical of deflection and frequency results for this class of wing structures

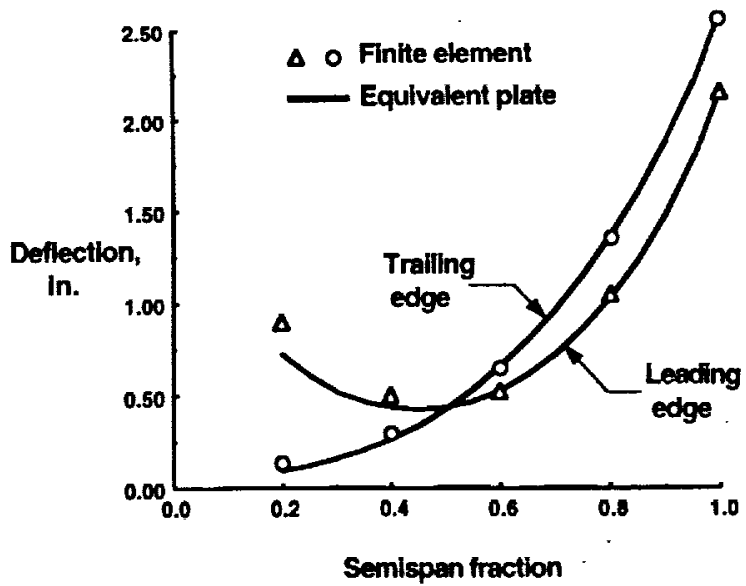

Figure 4. Thermal deflection of example wing.

The spanwise bending stress in the wing cover is shown in Figure 5 for the wing chord location that is indicated in Figure 3. Here, the maximum error in stress is approximately $20 \%$.

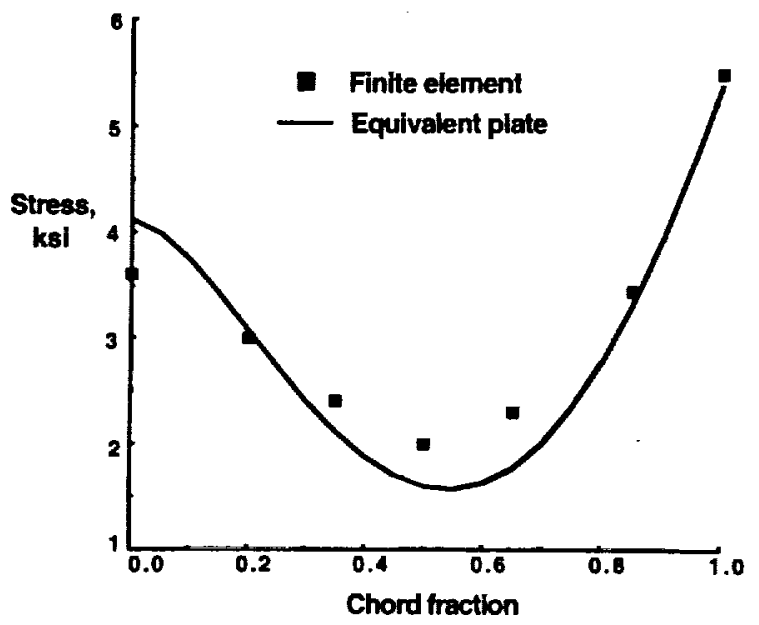

Figure 5. Comparison of stresses for example wing.

This level of accuracy was typical of stress results at other locations on wing which were presented in Reference [7]. The typical levels of accuracy from a variety of such applications are summarized in Figure 6 along with the speedup factor in computation time. These results indicate that levels of accuracy approaching that of a finite element analysis can be achieved by ELAPS in significantly less computation time. Hence, for wings in which bending behavior dominates, this formulation provides adequate accuracy for early preliminary design. 


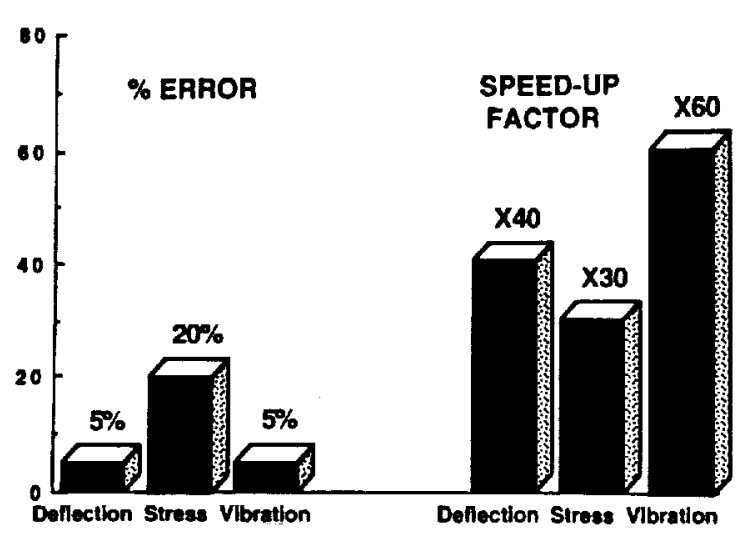

TYPE OF ANALYSIS

Figure 6 Summary of results from equivalent plate analysis compared to finite element analysis

ELAPS has also been used in studies of a high speed civil transport (HSCT). The wing structure for such a vehicle is designed for a lower load factor than for fighter aircraft, therefore transverse shear effects are likely to be important. Comparison of equivalent plate and finite results for the HSCT wing studied in Reference [8] indicated higher levels of error than for fighter wings. The deflections and stresses from both methods had similar distributions over the wing planform. However, the magnitude of the difference in wing tip deflection and maximum difference for the first 4 natural frequencies from the two methods was in the $10 \%$ range. Similarly, the stress results that were presented indicated a maximum difference of approximately $40 \%$. These levels of errors for HSCT wing is twice the level of errors for fighter wings and such levels have focused attention on the need to improve the accuracy of the equivalent plate analysis method. In another application, equivalent plate modeling was used to analyze a HSCT wing model being studied at the Boeing Company [9]. For the model used in this study, it was found that it was important to include the effects of transverse shear in the plate analysis formulation. The error in wing tip deflection compared to finite element analysis was reduced from approximately $20 \%$ when transverse shear was neglected to less that $10 \%$ when transverse shear was included. Similarly, the error in the eight lowest natural frequencies was reduced from a maximum of approximately $30 \%$ to less than $10 \%$ by including transverse shear. Such applications have indicated the need to add the capability for representing transverse shear effects in the ELAPS formulation. The modeling of discrete rib and spar shear webs would be included in such a formulation. Shear stresses in these webs could be calculated and could be used to size the web thicknesses. The weight of these webs could then be included along with the skin and caps weights in total weight calculations for the idealized structural model of a wing

\subsection{Coupling with Other Codes}

ELAPS can be easily coupled to other codes because its output quantities are calculated and expressed as continuous functions over the planform of the plate segments. These continuous functions can be evaluated exactly at a selected set of points on the analysis grid from any other code. The advantage of such direct coupling with ELAPS is the elimination of the cumbersome procedures that are often used for transforming data between non-matching analysis grids that are used by different disciplines. Two examples of coupling ELAPS with other codes will be presented.

In the first example, a coupled multiple-method structural analysis procedure is described [10]. Such a procedure could be used to evaluate wing structural designs by making changes to an equivalent plate model which is coupled to a fixed finite element model of a fuselage as shown in Figure 7. The equivalent plate and the finite element analysis methods are implemented in an integrated multiple-method formulation which involves the assembly and solution of a combined set of linear equations

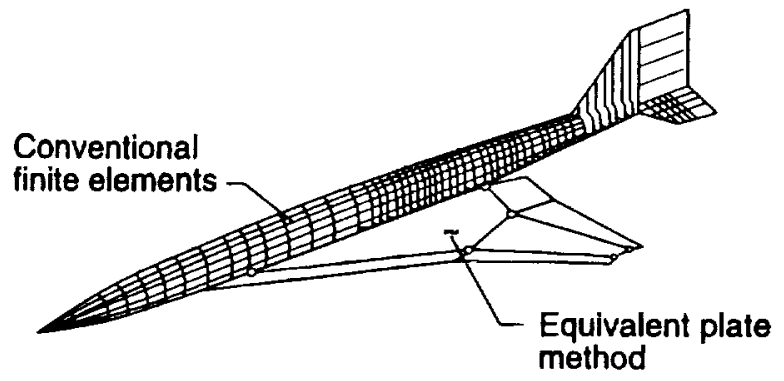

Figure 7. Coupled multiple-method structural analysis.

Two alternative approaches for coupling the methods were investigated; the first using transition finite elements and the second using Lagrange multipliers. The first approach required considerable code modification in that the two analysis had to be combined into a single code and transition finite elements had to be added to connect the two different analytical models. These transition elements had conventional finite element degrees of freedom (translations and rotations) at specified joints and the deformations of the other joints were defined in terms of the unknown polynomial coefficients. In the Lagrange multiplier approach, additional constraint equations were formed to equate the degrees of freedom of all attached finite element joints to corresponding deformations of the equivalent plate. The Lagrange multiplier approach required minimal code modification to implement since the stiffness matrices for both analysis methods could be generated and assembled in their original form using separate, unmodified analysis codes. Then these matrices were combined and solved using a third, smal! computer code which also generated the constraint equations to couple the analytical models. In Reference [10], the multiplemethod analysis procedure was used to perform structural analysis of a conceptual design representation in which the wing structure was modeled by the equivalent plate shown in Figure 3 and the fuselage and wing store/pylon structure were modeled using beam finite elements. Typically, static deflections and natural vibration frequencies and mode shapes from the coupled analysis method agreed within $5 \%$ to comparable results from a model composed of all finite elements. The stress results agreed within $20 \%$ as was typical of earlier studies, except in the area where the finite element representation of the store/pylon was attached to the equivalent plate wing model. In this area the difference was as large as $40 \%$ since the polynomial displacement functions in ELAPS are formulated to represent the global response of the 
wing and hence do not give adequate representation of stress concentrations in areas of localized load introduction

In the second example, equivalent plate models have been coupled with computational fluid dynamics (CFD) codes to perform static aeroelastic analyses early in the design process before a finite element structural model was available [11]. A direct, simple procedure to apply the aerodynamic loads to the equivalent plate model is facilitated by the continuous definition of the wing deflection. This procedure is shown schematically in Figure 8 . The equivalent load vector is composed of a set of equivalent forces which correspond to each term in the polynomial displacement function.

\section{Direct, simple application of loads}

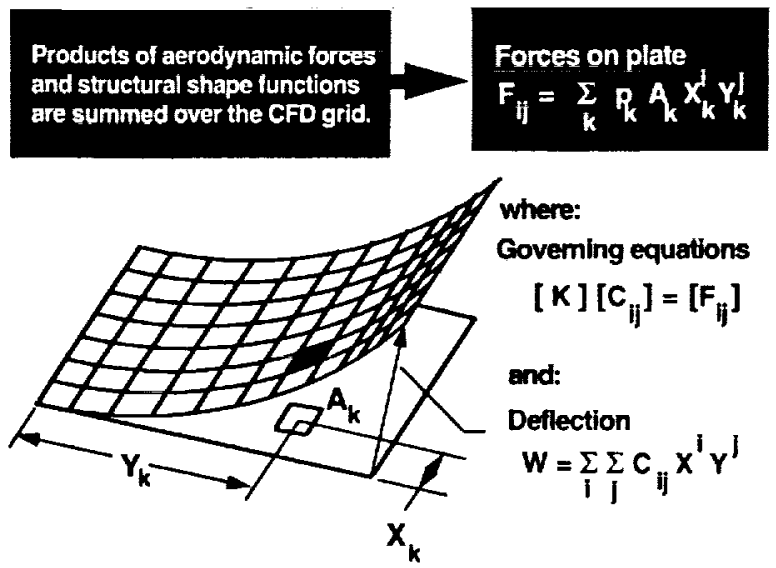

Figure 8. Procedure to couple CFD codes with equivalent plate analysis.

The first step in evaluating these equivalent forces is to form a set of aerodynamic forces as the product of the elemental surface area and pressure associated with each point on the CFD grid. In the second step, this set of aerodynamic forces is multiplied by the non-dimensional portion (shape function) of a particular term from the displacement function which has been evaluated at the location of the aerodynamic force. This evaluation is simple and direct since the polynomial displacement functions are defined as continuous functions over the entire wing planform. These resulting products are summed for all forces on the aerodynamic grid. This second step is repeated for each term in the polynomial displacement function in order to form the entire equivalent load vector

ELAPS was used for structural modeling in a design study to investigate the effects of nonlinear aerodynamics and static aeroelasticity on mission performance calculations for an advanced fighter aircraft [12]. The wing structure of this vehicle was relatively stiff. Three different aerodynamic codes (linear, full potential and Euler) with different levels of accuracy were used in the study as indicated in Figure 9. Aeroelastic calculations were performed only using the CFD codes (full potential and Euler). Vehicle performance, shown in terms of total mission radius, was found to be influenced more by the choice of aerodynamic theory used than by the aeroelastic effects for this stiff wing.However, this is an example of the variable complexity modeling approach [13] which could be effective in design of flexible wings where the sophistication of CFD codes might be needed for aerodynamic design and where the structural flexibility effects could be adequately represented with the more approximate equivalent plate analysis. The relatively small size of the ELAPS code offers the practicality of embedding it directly into the iterative procedure of a CFD code so that the combined code would converge to an aerodynamic solution on a deformed shape with a minimal increase in computational time over that required for the analysis of a rigid shape

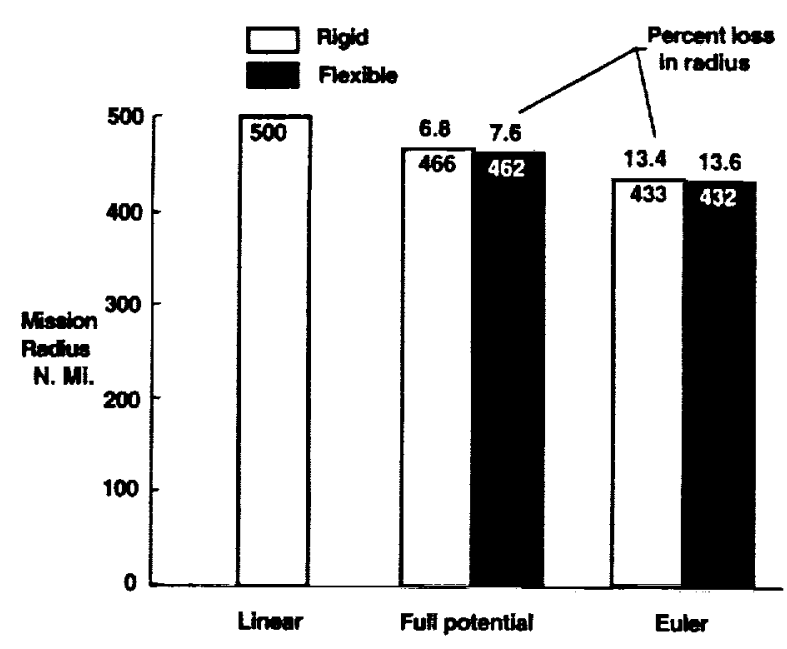

Figure 9. Effects of different aerodynamic theories and structural flexibility on mission performance.

\subsection{Sensitivity Derivatives}

The capability to calculate sensitivity derivatives is an important feature of a design oriented analysis method. One approach that is often used is referred to as the finite difference method in which repeated analyses are performed as the values of the design variables are systematically perturbed. A shortcoming of this method is that loss of accuracy can result from improper selection of the magnitude (step size) of the perturbation. A second approach, referred to as analytical or quasi-analytical, is to differentiate to governing equations and to implement the resulting expressions in a new code. This approach is time-consuming and error-prone since it involves a new formulation and coding which must be thoroughly debugged and verified.

The capability to generate sensitivity derivatives has been provided in ELAPS through use of the recently developed ADIFOR (Automatic Differentiation of FORTRAN) code [14]. ADIFOR is a pre-compiler tool which augments existing analysis codes with sensitivity derivative calculations for user specified output quantities with respect to specified input variables. Automatic differentiation is based on computing derivatives of all elementary operations contained in each line of the original analysis code and applying the chain rule over and over again to compute exact, desired derivative information. Since ADIFOR is a pre-compiler tool, it can be readily applied to a broad range of applications by both aerospace and nonaerospace industries. This procedure has been used with ELAPS to provide for derivatives of 16,500 strains and stresses with respect to 44 structural design variables during design studies of a HSCT wing structure [15]. The results with ADIFOR show a reduction of up to $80 \%$ of the computer time with respect to a simple finite difference process amounting to 
rerunning the basic analysis code, once for the baseline analysis and once for each independent design variable.

\subsection{Sensitivity-Based Scaling}

Design oriented analysis methods generally produce results which are approximations to results that would be produced by a refined analysis method. Scaling factors have been used in the past for the correlation of simplified and refined models. The use of a scale factor involves comparing results from an approximate model or method at a given design point, to results from a more refined model or method. The ratio of results from the refined method to results from the approximate method is a scaling factor that is used to multiply the approximate results at other points in the design domain.

A sensitivity-based scaling approach [16] has been developed and used with ELAPS which refines the conventional constant scaling factor by using a linearly varying scale factor. This approach is based on differentiating the conventional scale factor with respect to a selected design variable and forming an expression for a scale factor which varies linearly as that particular design variable is changed. This expression requires sensitivity derivatives from both the approximate and refined analysis methods.

This sensitivity-based scale factor was used to correlate structural response from the approximate ELAPS code and a refined representation using a conventional finite element analysis code in Reference [16]. A typical result from this study is shown in Figure 10.

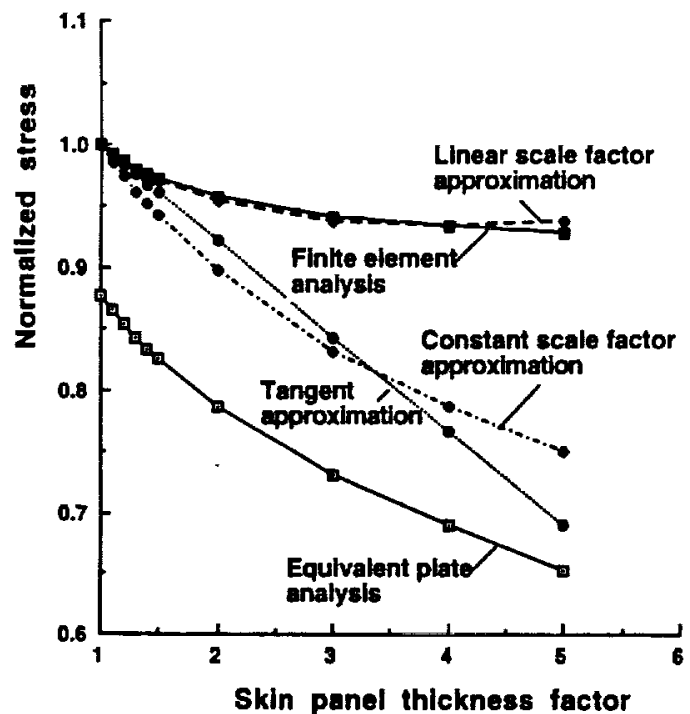

Figure 10. Approximations to representative von Mises stress.

A representative von Mises stress was calculated as a skin panel thickness was changed up to a factor of five thicker than the original skin using both the ELAPS equivalent plate analysis method and a finite element analysis method. Then, three approaches were compared for approximating the change in stress as the skin thickness was changed. The conventional tangent approximation and constant scale factor are shown to approximate the finite element results within a 10 percent error if the thickness change is limited to a factor of two. The linear scale factor is shown to give good correlation (within 1 percent error) with the refined analysis results over a considerable range of change in design variable (up to a factor of 5 change). This approach permits an efficient, approximate code such as ELAPS to be used in iterative optimization procedures in which the accuracy of results are retained by periodically updating the linear scale factor at selected cycles in the optimization process. Hence, sensitivity-based scaling offers an approach to provide adequate accuracy for early preliminary design in applications where approximations in the design oriented analysis methods may not permit their analysis data to be used directly in the design process.

\subsection{Multidisciplinary Design Application}

ELAPS has been applied in a variety of design studies. A particular example is its use in a multidisciplinary design integration system for conducting design methodology research and for performing design studies of high speed civil transport configurations at the NASA Langley Research Center. This system is referred to as the High Speed Airframe Integration Research (HiSAIR)/Pathfinder system [17,18]. This system, shown schematically in Figure 11 , is representative of multidisciplinary optimization tools. The disciplines of mission performance, aerodynamics and structures are included in this Pathfinder system

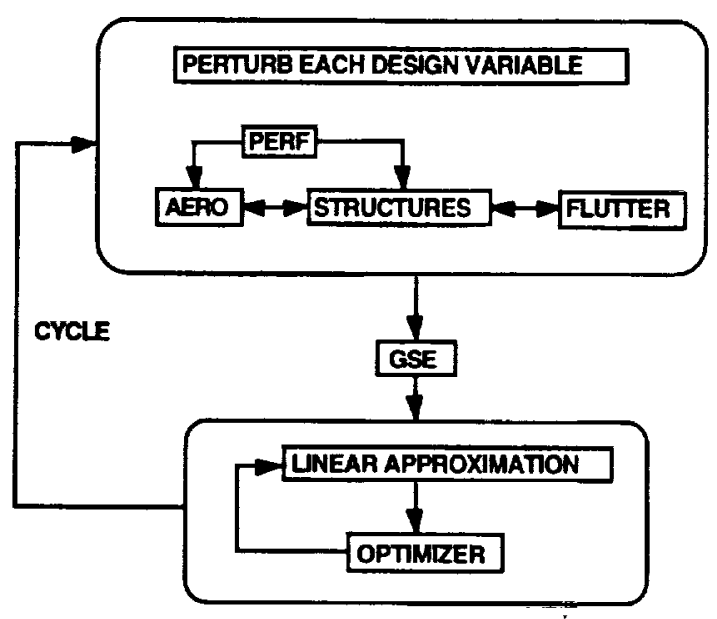

Figure 11. Flow chart of HiSAIR/Pathfinder optimization system.

The Generalized Sensitivity Equation (GSE) approach [19] is used to combine partial sensitivity derivatives from each of the disciplines into total derivatives for use by the optimizer. Structural analysis is performed by ELAPS

In a design study described in Reference [18], the goal was to resize the wing cover panels and internal structure for minimum mass. Forty-four (44) independent design variables were used to control the cross-sectional areas of wing rib and spar caps and the thicknesses of wing skin cover panels as indicated in Figure 12. A total of 300 stress, strain, buckling and displacement constraints and minimum gages were used to optimize the idealized wing structure. A typical optimization convergence history that was obtained during this design study is shown in Figure 13. A total of 180 ELAPS analysis runs were required to reach the converged design. This experience indicates that the computational efficiency of ELAPS is an 
important advantage for direct use in optimization studies. An alternate approach, would be to use ELAPS to perform the large number of analyses that are necessary to generate response surfaces [20] for use in vehicle system studies. The design study discussed in this section demonstrates that ELAPS contains a combination of desirable characteristics for a design oriented analysis which makes it effective for use in a multidisciplinary design optimization system

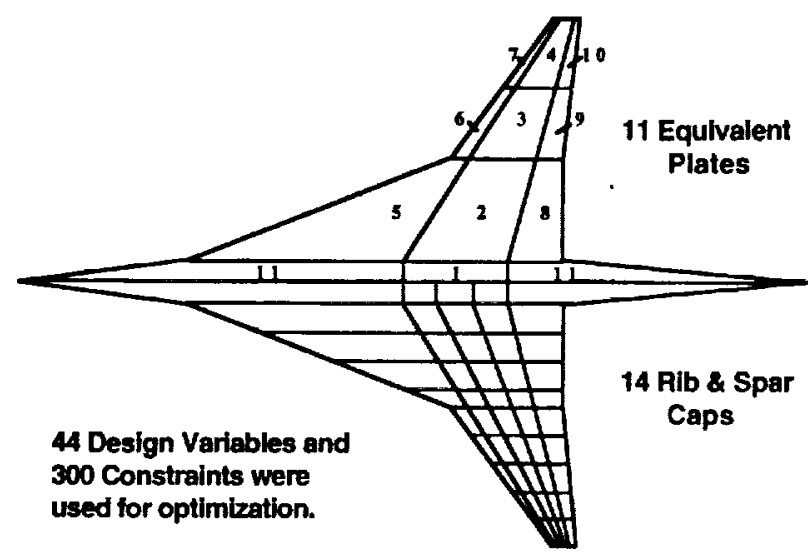

Figure 12. Structural model used in HSCT design studies

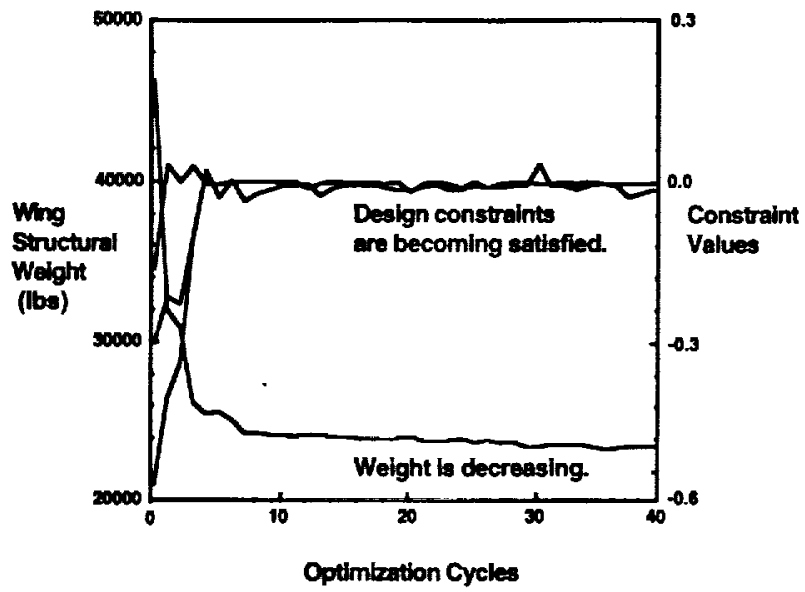

Figure 13. Optimization convergence history.

\section{Related Developments}

The equivalent plate approach has been used to perform sensitivity analysis of wing static aeroelastic response and wing flutter with respect to planform shape variables. Both an iterative and $a$ direct solution procedure were used for performing such calculations with linear aerodynamics in Reference [21]. The shape variables considered were wing sweep, aspect ratio, wing area and taper ratio. Considerable attention was given to establishing an accurate, efficient procedure to interface the aerodynamic analysis to the structural analysis. Initially, Chebyshev polynomials were used to represent the pressure distribution, in a global sense, over the entire wing planform. Improved accuracy was obtained using a piecewise linear representation since it would capture small, local details in the pressure field. In this study, the ELAP $\bar{S}$ code was used to perform structural analysis. In a subsequent study to calculate sensitivity of flutter response [22], Chebyshev polynomials were used to represent the deflection of the wing structure instead of the power series polynomials that are used in ELAPS. The orthogonal nature of the Chebyshev polynomials made it possible to include higher powers in the polynomials that were use to describe the wing deflection and thus better represent the flutter behavior of a wing. The shape sensitivity derivatives of the flutter speed of a wing using an analytical method and using finite difference calculations were found to be in good agreement.

Another equivalent plate analysis code, LS-CLASS $[23,24]$, which in similar to ELAPS, was developed for aeroservoelastic applications and has been used to carry out structural/aerodynamic/control optimization of fiber composite actively controlled wings in an integrated manner. The LSCLASS code is based on classical plate theory which neglects transverse shear. Subsequently, an improved formulation which includes the kinematic assumptions of first order shear deformation has been implemented [25]. The capabilities to calculate analytical derivatives of stiffness, mass and load terms with respect to wing shape design variables are contained in the new code. Analytical sensitivity derivatives of displacements, stresses and natural modes and frequencies with respect to planform shape and wing depth distribution can then be calculated, thereby providing an effective structural tool for wing shape optimization

\section{Concluding Remarks}

Desirable features of design oriented analysis methods are discussed. These methods are intended to provide the capability for rapid and reliable assessment of the effects of proposed design changes during early phases of design before the external shape of the vehicle is fixed. These features include; adequate accuracy for early preliminary design, efficient computation, capability to trade accuracy for speed, minimal time for model preparation and modification, capability to generate sensitivity derivatives, ease of coupling with other codes and effective for use in multidisciplinary design optimization systems. These desirable characteristics and benefits are illustrated for the structures discipline by presenting an overview description of the development of equival ent plate analysis methods. The features and uses of the ELAPS code are emphasized in this discussion. This code is shown to contain a combination of these features which makes it effective for generating data needed during early preliminary design. Such design oriented analysis capabilities offer significant potential for reducing design cycle time and/or resulting in an improved product design.

Examples were described in the paper illustrating a variety of uses for ELAPS during conceptual or early preliminary design of wing structures. The accuracy and efficiency of results from ELAPS analyses were compared with results from finite element analyses. Ease of coupling ELAPS with CFD codes to calculate aeroelastic effects early in the design process before a finite element model is available was illustrated. The reduced computational time obtained from use of the ADIFOR pre-complier with ELAPS to produce code for calculation of 
sensitivity derivatives was discussed. It was demonstrated that the accuracy of finite element analyses could be approximated over a wide range of design variable change by applying a linear scale factor to ELAPS results. Finally, use of ELAPS in a multidisciplinary design optimization system for performing design studies of high speed civil transport configurations was described.

\section{References}

1. McCullers, L.A. (1984) "Aircraft Configuration Optimization Including Optimized Flight Profiles", NASA CP-2327, Proceedings of Symposium on Recent Experiences in Multidisciplinary Analysis and Optimization, 395-412

2. Sobieszczanski-Sobieski, J. (1993) "Multidisciplinary Design Optimization: An Emerging New Engineering Discipline", Presented at The World Congress on Optimal Design of Structural Systems, Rio de Janeiro, Brazil

3. McCullers, L.A., Lynch, R.W. (1974) "Dynamic Characteristics of Advanced Filamentary Composite Structures", AFFDL-TR-73-111, Vol. Il

4. Lynch, R.W., Rogers, W.A., Braymen, W.W. (1976) "An Integrated Capability for the Preliminary Design of Aeroelastically Tailored Wings", AIAA Paper 76-912

5. Triplett, W.E. (1979) "Aeroelastic Tailoring Studies in Fighter Aircraft Design", AIAA Paper 79-0725.

6. Giles, G.L. (1986) "Equivalent Plate Analysis of Aircraft Wing Box Structures with General Planform Geometry", J. of Aircraft, Vol. 23, No. 11, 859-864.

7. Giles, G.L. (1989) "Further Generalization of an Equivalent Plate Representation for Aircraft Structural Analysis", J. of Aircraft, Vol. 26, No. 1, 67-74.

8. Kao, P.-J., Wrenn, G.A., and Giles, G.L. (1990) "Comparison of Equivalent Plate and Finite Element Analysis of a Realistic Aircraft Structural Configuration", AIAA Paper No. 90-3293, AIAA/AHS/ASEE Aircraft Design Systems and Operations Conference, Dayton, $\mathrm{OH}$

9. Livne, E., Sels, R.A., Bhatia, K.G. (1993) "Lessons from Application of Equivalent Plate Structural Modeling of an HSCT Wing", AIAA Paper No. 93-1413-CP, 34th AIAA/ASME/ASCE/AHS/ASC Structures, Structural Dynamics, and Materials Conference, La Jolla, CA, 959. 969

10. Giles, G.L., Norwood, R.K. (1990) "Coupling Equivalent Plate and Finite Element Formulations in Multiple-Method Structural Analyses", Presented at the Third Air Force/NASA Symposium on Recent Advances in Multidisciplinary Analysis and Optimization, San Francisco, CA. Submitted for publication in $J$. of Aircraft.
11. Tatum, K.E., Giles, G.L. (1988) "Integrating Aerodynamic and Structural Analysis for a Complete Fighter Configuration", J. of Aircraft, Vol. 25, No. 12, 1150-1156

12. Giles, G.L., Tatum, K.E., Foss, W.E.Jr. (1988) "Effects of Nonlinear Aerodynamics and Static Aercelasticity on Mission Performance Calculations for a Fighter Aircraft", Presented at the Second NASA/Air Force Symposium on Recent Advances in Multidisciplinary Analysis and Optimization, Hampton, VA.

13. Unger, E.R., Hutchinson, M.G., Rais-Rohani, M., Haftka, R. T., Grossman, B. (1992) "Variable-Complexity Multidisciplinary Design of a Transport Wing", International Journal of Systems Automation: Research and Applications (SARA) 2, 87-113

14. Bischof, C., Griewank A., "Computational Differentiation and Multidisciplinary Design", Argonne Preprint MCSP420-0294, to appear in Proceedings of the Symposium on Inverse Problems and Optimal Design in Industry, J. McLaughlin and H. Engl, Eds., Teubner Verlag, Stuttgart, Germany, 1994

15. Barthelemy, J.-F.M., Hall, L.E. (1992) "Automatic Differentiation as a Tool for Engineering Design", Presented at the 4th AIAA/USAF/NASA/OAI Symposium on Multidisciplinary Analysis and Optimization, Cleveland, $\mathrm{OH}$. Submitted for publication in Structural Optimization.

16. Chang, K.J., Haftka, R.T., Giles, G.L., Kao, P.-J. (1993) "Sensitivity-Based Scaling for Approximating Structural Response", J. of Aircraft, Vol. 30, No. 2, 283-288.

17. Barthelemy, J.-F.M., Wrenn, G.A., Dovi, A.R., Coen, P.G., Hall, L.E. (1994) "Supersonic Transport Wing Design Integrating Aerodynamics and Structures", J. of Aircraft, Vol. 31, No. 2, 330-338.

18. Dovi, A.R., Wrenn, G.A., Barthelemy, J.M., Coen, P.G., Hall, L.E. (1992) "Multidisciplinary Design Integration System for a Supersonic Transport Aircraft", Presented at the 4th AIAAUSAF/NASA/OAI Symposium on Multidisciplinary Analysis and Optimization, Cleveland, $\mathrm{OH}$.

19. Sobieszczanski-Sobieski, J. (1990) "Sensitivity of Complex Intemally Coupled Systems", AIAA Joumal, Vol 28 , No. 1, 153-160.

20. Carpenter, W., Barthelemy, J. (1992) "A Comparison of Polynomial Approximations and Artificial Neural Nets as Response Surfaces", AIAA Paper No. 92-2247-CP, 33th AIAA/ASME/ASCE/AHS/ASC Structures, Structural Dynamics, and Materials Conference, Dallas, TX, 24742482

21. Eldred, L.B., Kapania, R.K., Barthelemy, J.-F.M. (1993) "Sensitivity Analysis of Aeroelastic Response of a Wing Using Piecewise Pressure Representation". AIAA Paper No. 93-1645-CP, 34th AIAA/ASME/ASCE/AHS/ASC Structures, Structural Dynamics, and Materials Conference, La Jolla, CA, 2974-2984. 
22. Kapania, R.K., Issac, J.C., Barthelemy, J.-F.M. (1993) "Sensitivity Analysis of Flutter Response of a Typical Section and a Wing in Transonic Flow", AIAA Paper No. 93-1646-CP, 34th AIAA/ASME/ASCE/AHS/ASC Structures, Structural Dynamics, and Materials Conference, La Jolla, CA, 2985-2997.

23. Livne, E., Schmit, L.A., Friedmann, P.P. (1988) "Design Oriented Structural Analysis for Fiber Composite Wings", UCLA Report UCLA-ENG-88-36, Department of Mechanical, Aerospace and Nuclear Engineering, University of California, Los Angeles CA.
24. Livne, E. (1990) "Integrated Multidisciplinary Optimization of Actively Controlled Fiber Composite Wings", Ph.D. Dissertation, Department of Mechanical, Aerospace and Nuclear Engineering, University of California, Los Angeles CA

25. Livne, E. (1993) "Recent Developments in Equivalent Plate Modeling for Wing Shape Optimization", AIAA Paper No. 93-1647, 34th AIAA/ASME/ASCE/AHS/ASC Structures, Structural Dynamics, and Materials Conference, La Jolla, CA, 2998-3011 


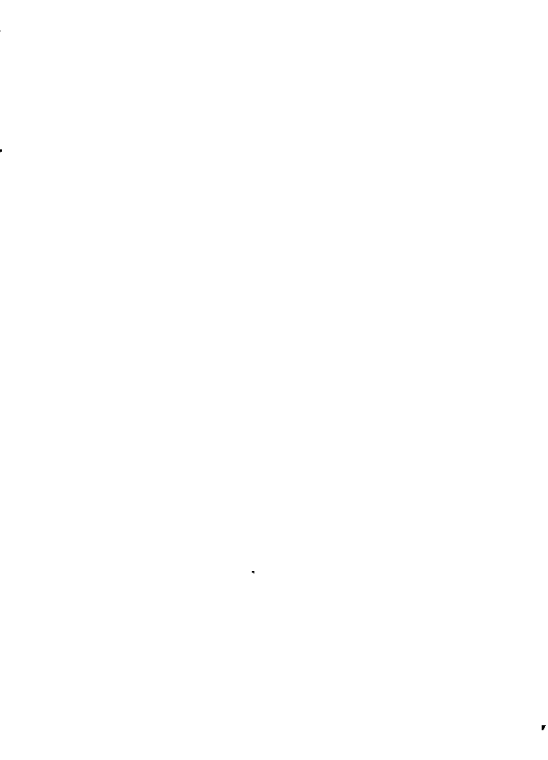

$=$

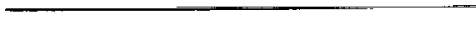

,

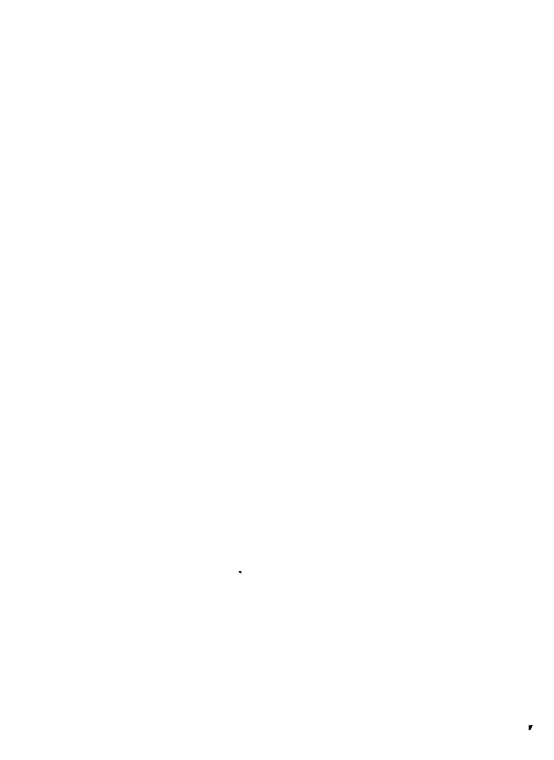

.

.

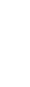

(2)

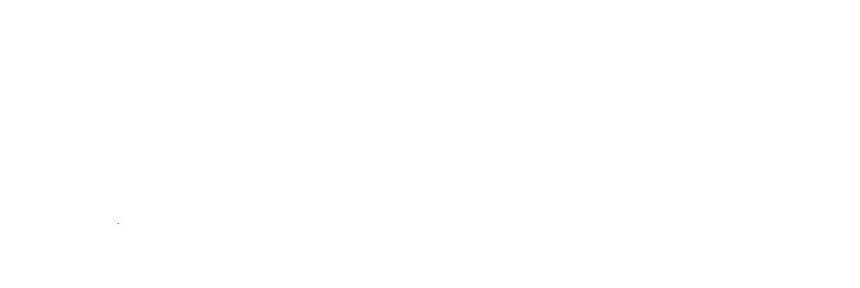




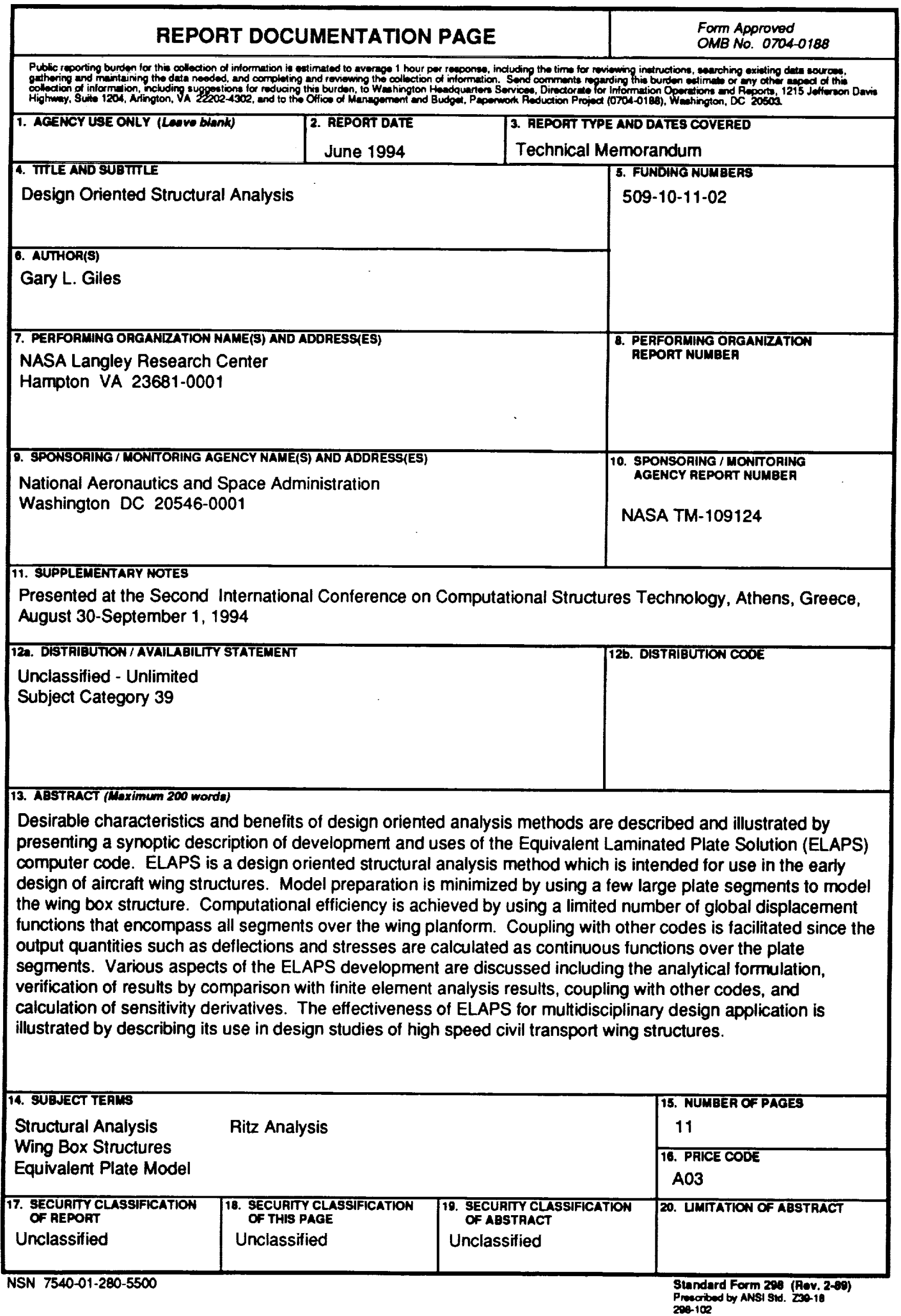

